\title{
Riemann-Liouville Fractional Calculus of Blancmange Curve and Cantor Functions
}

\author{
Srijanani Anurag Prasad \\ Department of Mathematics and Statistics, Indian Institute of Technology Tirupati, India.
}

\begin{abstract}
How to cite this paper: Srijanani Anurag Prasad. (2020) Riemann-Liouville Fractional Calculus of Blancmange Curve and Cantor Functions. Journal of Applied Mathematics and Computation, 4(4), 123-129. DOI: $10.26855 /$ jamc.2020.12.003
\end{abstract}

Received: September 15, 2020

Accepted: October 10, 2020

Published: October 22, 2020

*Corresponding author: Srijanani Anurag Prasad, Department of Mathematics, Indian Institute of Technology Tirupati, India.

Email: srijanani@iittp.ac.in

\begin{abstract}
Riemann-Liouville fractional calculus of Blancmange Curve and Cantor Functions are studied in this paper. In this paper, Blancmange Curve and Cantor function defined on the interval is shown to be Fractal Interpolation Functions with appropriate interpolation points and parameters. Then, using the properties of Fractal Interpolation Function, the Riemann-Liouville fractional integral of Blancmange Curve and Cantor function are described to be Fractal Interpolation Function passing through a different set of points. Finally, using the conditions for the fractional derivative of order $v$ of a FIF, it is shown that the fractional derivative of Blancmange Curve and Cantor function is not a FIF for any value of $v$.
\end{abstract}

\section{Keywords}

Fractal, Interpolation, Iterated Function System, fractional integral, fractional derivative, Blancmange Curve, Cantor function

\section{Introduction}

Fractal geometry is a subject in which irregular and complex functions and structures are researched. Fractal Interpolation Function (FIF) was introduced by Barnsley using the theory of Iterated Function System (IFS) [1]. Different kinds of FIFs like Hidden-variable FIFs, Coalescence Hidden-variable FIFs, Hermite FIFs, Spline FIFs, Super FIFs have been constructed [2-7].

Unlike classical Newtonian derivatives, the Riemann-Liouville fractional derivative is defined via fractional integral. A lot of works on fractional calculus already exist in the literature [8-11]. Fractional calculus of a FIF has been investigated in [12, 13], and Fractional calculus of a CHFIF was explored [14]. In this paper, Blancmange Curve or Takagi Curve and Cantor function is first shown to be a Fractal Interpolation Function with an appropriate number of interpolation points and relevant parameters. Then, the $v$-order Riemann-Liouville fractional integral of Blancmange Curve and Cantor function is derived. Further, using the conditions for the fractional derivative of order $v$ of a FIF, it is shown that the fractional derivative of Blancmange Curve and Cantor function is not a FIF for any value of $v$.

The organization of the paper is as follows: Section 2 starts with a brief introduction to the construction of a Fractal Interpolation Function (FIF). A FIF is constructed as the graph of the attractor of a suitably defined Iterated Function System (IFS). The definition of $v$-order Riemann-Liouville fractional integral and definition of $v$-order Riemann-Liouville fractional derivative is given in Section 3. Then, the generalized results of the fractional integral of FIF on any interval [a, b] are presented. Also, properties relating fractional derivative and fractional integral and the condition for v-order Riemann-Liouville fractional derivative of FIF are also discussed in the same section. In Section 4 and Section 5, it is first shown that Blancmange Curve and Cantor function can be viewed as a Fractal Interpolation Function. Then, the fractional integral of order $v$ of Blancmange Curve and Cantor function are derived using the generalized results. Further, using the conditions for the fractional derivative of order $v$ of a FIF, it is shown that the fractional derivative of Blancmange Curve and Cantor function is not a FIF for any value of $v$. 


\section{Construction of Fractal Interpolation Function (FIF)}

This section begins with a brief introduction of Fractal Interpolation Function (FIF), which is constructed such that it is a fixed point of Read-Bajraktarevic operator on the suitable space of continuous functions.

Given any interpolation data $\left\{\left(x_{i}, y_{i}\right) \in \mathbb{R}^{2}: i=0,1, \ldots, N\right\}$, where $-\infty<x_{0}<x_{1}<\ldots<x_{N}<\infty$, the largest interval $\left[x_{0}, x_{N}\right]$ and the smaller sub-intervals $\left[x_{n-1}, x_{n}\right]$ for $n=1,2, \ldots, N$ are denoted by $I$ and $I_{n}$ respectively. For $n=1,2, \ldots, N$, the contraction maps $L_{n}$ from the interval $I$ into subintervals $I_{n}$ are defined as

$$
L_{n}(x)=a_{n} x+b_{n}=x_{n-1}+\frac{x_{n}-x_{n-1}}{x_{N}-x_{0}}\left(x-x_{0}\right) .
$$

Suppose the functions $F_{n}: I \times \mathbb{R} \mapsto \mathbb{R}$ for $n=1,2, \ldots, N$ are defined as

$$
F_{n}(x, y)=\gamma_{n} y+q_{n}(x)
$$

where, $\gamma_{n}$ are chosen such that $\left|\gamma_{n}\right|<1$ and $q_{n}$ are continuous functions chosen such that $F_{n}\left(x_{0}, y_{0}\right)=y_{n-1}$ and $F_{n}\left(x_{N}, y_{N}\right)=y_{n}$ are satisfied. The variables $\gamma_{n}$ are called free variables, and the above condition is called join-up conditions. The suitable Iterated Function System (IFS) required to construct a Fractal Interpolation Function (FIF) is now defined as

$$
\left\{I \times \mathbb{R} ; \omega_{n}, n=1,2, \ldots N\right\}
$$

where, $\omega_{n}(x, y)=\left(L_{n}(x), F_{n}(x, y)\right)$. It was proved by Barnsley [1] that the IFS constructed with the above chosen free parameters and satisfying the join up conditions is a hyperbolic IFS for a metric $d^{*}$ on $\mathbb{R}^{2}$ equivalent to the Euclidean metric. Therefore, on $H\left(\mathbb{R}^{2}\right)$, the space of compact sets in $\mathbb{R}^{2}$, there exists a unique set $A$ such that $\quad A=\bigcup_{k=1}^{N} \omega_{k}(A)$, where $\omega_{k}(A)=\left\{\omega_{k}(x, y):(x, y) \in A\right\}$. This fixed set $A$ is called the attractor of the above hyperbolic IFS constructed with the given interpolation data. Barnsley showed in the same paper that this attractor is graph of a continuous function $f: I \rightarrow \mathbb{R}$ such that $f\left(x_{i}\right)=y_{i}$ for $i=0,1, \ldots, N$, and thus Fractal Interpolation Function (FIF) was defined as:

The Fractal Interpolation Function (FIF) for the interpolation data $\left\{\left(x_{i}, y_{i}\right): i=0,1, \ldots, N\right\}$ is defined as the continuous function $f: I \rightarrow \mathbb{R}$, whosegraph is the attractor of IFS $\left\{I \times \mathbb{R} ; \omega_{n}, n=1,2, \ldots, N\right\}$.

Consider the space $\left(\mathfrak{I}, d_{\mathfrak{J}}\right)$, where the set $\mathfrak{I}$ is defined by $\mathfrak{I}=\left\{g \mid g: I \rightarrow \mathbb{R}\right.$ is continuous, $g\left(x_{0}\right)=$ $y_{0}$ and $\left.g\left(x_{N}\right)=y_{N}\right\}$ and the maximum metric $d_{\mathfrak{I}}$ on the set, $\mathfrak{I}$ is given by $d_{\mathfrak{I}}(g, \hat{g})=\max _{x \in I}|g(x)-\hat{g}(x)|$. It was shown in [1] that FIF $f$, is a fixed point of Read-Bajraktarevic operator $T$ defined by, $T(g)(x)=F_{n}\left(L_{n}^{-1}(x), g\left(L_{n}^{-1}(x)\right)\right), x \in I_{n}$. Hence, the FIF $f$ satisfies the recursive equation

$$
f\left(L_{n}(x)\right)=F_{n}(x, f(x)), \quad x \in I \text { and } n=1,2, \ldots, N
$$

where, $L_{n}$ and $F_{n}$ are as defined in (1) and (2). So, another way to define Fractal Interpolation Function (FIF) is as follows:

The Fractal Interpolation Function (FIF) for the interpolation data $\left\{\left(x_{i}, y_{i}\right): i=0,1, \ldots, N\right\}$ is defined as the continuous function $f: I \rightarrow \mathbb{R}$ which satisfies the recursive equation given by (4).

\section{Fractional Calculus of FIF}

In this section, we start with the definition of Riemann-Liouville fractional integral of order $v>0$ of a function. Then, we shall recall the fact that Riemann-Liouville fractional integral of a FIF is a FIF, which is proved in [12-14]. Next, we shall define Riemann-Liouville fractional derivatives of order $v>0$ of a function. Finally, the condition under which the $v$-order Riemann-Liouville fractional derivative of a FIF exist is also given.

Let $-\infty<a<x<b<\infty$. The Riemann-Liouville fractional integral of order $v>0$ with lower limit $a$ is defined for locally integrable functions $f:[a, b] \rightarrow \mathbb{R}$ as $I_{a+}^{v} f(x)=\frac{1}{\Gamma(v)} \int_{a}^{x}(x-t)^{v-1} f(t) d t$. For $v=0$, it is defined that $I_{a+}^{v} f(x)=f(x)$.

The following proposition is proved in [12-14] and shows that the $v$-order Riemann-Liouville fractional integral of a FIF defined on any interval $[a, b] \subset \mathbb{R}$ is a FIF.

Proposition 1: Let $f$ be a FIF passing through the interpolation data given by $\left\{\left(x_{i}, y_{i}\right) \in \mathbb{R}^{2}: i=0,1, \ldots, N\right\}$ and constructed using the IFS given by (3). Define $F_{n}^{v}(x, y)=a_{n}^{v} \gamma_{n} y+q_{n}^{v}(x)$ where,

$$
q_{n}^{v}(x)=a_{n}^{v} I_{x_{0}}^{v} q_{n}(x)+\frac{1}{\Gamma(v)} \int_{x_{0}}^{x_{n-1}}\left(L_{n}(x)-t\right)^{v-1} f(t) d t .
$$

Then, Riemann-Liouville fractional integral of a FIF of order $v$ is also a FIF passing through the data $\left\{\left(x_{i}, y_{i}^{v}\right) \in \mathbb{R}^{2}:\right.$ $i=0,1, \ldots, N\}$, where $y_{0}^{v}=0, y_{N}^{v}=\frac{q_{N}^{v}\left(x_{N}\right)}{1-a_{N}^{v} \gamma_{N}}$, and $y_{j}^{v}=a_{j}^{v} \gamma_{j} y_{N}^{v}+q_{j}^{v}\left(x_{N}\right)=q_{j+1}^{v}\left(x_{0}\right)$ for $j=1, \ldots, N-1$. 
Proof: For any $x \in\left[x_{0}, x_{N}\right]$ and $k \in\{1,2, \ldots, N\}$,

$$
\begin{aligned}
I_{x_{0}}^{v} f\left(L_{k}(x)\right)= & \frac{1}{\Gamma(v)} \int_{x_{0}}^{L_{k}(x)}\left(L_{k}(x)-t\right)^{v-1} f(t) d t \\
& =\frac{1}{\Gamma(v)}\left\{\int_{x_{0}}^{x_{k-1}}\left(L_{k}(x)-t\right)^{v-1} f(t) d t+\int_{x_{k-1}}^{L_{k}(x)}\left(L_{k}(x)-t\right)^{v-1} f(t) d t\right\}
\end{aligned}
$$

Replacing $t$ by $L_{k}(t)$ in the second integral and using $f\left(L_{k}(t)\right)=\gamma_{k} f(t)+q_{k}(t)$ for all $k=1,2, \ldots, N$, it follows that

$$
\begin{aligned}
I_{x_{0}}^{v} f\left(L_{k}(x)\right)= & \frac{1}{\Gamma(v)}\left\{\int_{x_{0}}^{x_{k-1}}\left(L_{k}(x)-t\right)^{v-1} f(t) d t+\int_{x_{0}}^{x}\left(L_{k}(x)-L_{k}(t)\right)^{v-1} f\left(L_{k}(t)\right) a_{k} d t\right\} \\
= & \frac{1}{\Gamma(v)}\left\{\int_{x_{0}}^{x_{k-1}}\left(L_{k}(x)-t\right)^{v-1} f(t) d t+a_{k}^{v} \int_{x_{0}}^{x}(x-t)^{v-1}\left[\gamma_{k} f(t)+q_{k}(t)\right] d t\right\} \\
= & a_{k}^{v} \gamma_{k} I_{x_{0}}^{v} f(x)+a_{k}^{v} I_{x_{0}}^{v} q_{k}(x)+\frac{1}{\Gamma(v)}\left\{\int_{x_{0}}^{x_{k-1}}\left(L_{k}(x)-t\right)^{v-1} f(t) d t\right\}
\end{aligned}
$$

Using (5), it follows $I_{x_{0} 0}^{v} f\left(L_{k}(x)\right)=a_{k}^{v} \gamma_{k} I_{x_{0}}^{v} f(x)+q_{k}^{v}(x)=F_{k}^{v}\left(x, I_{x_{0}}^{v} f(x)\right)$, which implies that $v$-order Riemann-Liouville fractional integral of the function $f$ is a FIF passing through the data $\left\{\left(x_{i}, y_{i}^{v}\right) \in \mathbb{R}^{2}: i=0,1, \ldots, N\right\}$.

Now, we define Riemann-Liouville fractional derivatives of order $v>0$ for a function $f \in L_{1}([a, b])$.

Let $-\infty<a<x<b<\infty, 0<v$ and $f \in L_{1}([a, b]$. Let

$W^{k, j}=\left\{f: f \in L_{j}([a, b])\right.$ and $\left.D^{(k)} f \in L_{j}([a, b])\right\}$ and $I^{n-v} f \in W^{1,1}$, where $n$ is the smallest integer greater than

$v$. The Riemann-Liouville fractional derivative of order $v$ with lower limit $a$ is defined as $\left(D_{a}^{v} f\right)(x)=\frac{d^{n}}{d x^{n}}\left(I_{a}^{n-v} f\right)(x)$ and $\left(D_{a}^{0} f\right)(x)=f(x)$.

The following proposition is proved in $[13,14]$ and gives the condition under which the $v$ - order Riemann-Liouville fractional derivative of a FIF exist.

Proposition 2:

Let $f$ be a FIF passing through the interpolation data given by $\left\{\left(x_{i}, y_{i}\right) \in \mathbb{R}^{2}: i=0,1, \ldots, N\right\}$ and constructed using the IFS given by (3). Suppose $\gamma_{k}<a_{k}^{v}$ for some fixed $v>0$. Then Riemann-Liouville fractional derivative of a FIF of order $v$ is also a FIF provided

$$
\begin{aligned}
q_{k}^{d v}(x)=a_{k}^{-v} D^{v} & q_{k}(x)+\frac{1}{\Gamma(n-v)} \frac{d^{n}}{d\left(L_{k}(x)\right)^{n}} \int_{x_{0}}^{x_{k-1}} f(t)\left(L_{k}(x)-t\right)^{n-v-1} d t \\
= & a_{k}^{-v} D^{v} q_{k}(x)+\frac{1}{\Gamma(n-v)}\left(\prod_{j=1}^{n}(n-j-v)\right) \int_{x_{0}}^{x_{k-1}} f(t)\left(L_{k}(x)-t\right)^{-v-1} d t
\end{aligned}
$$

Proof: Using the definition of Riemann-Liouville fractional derivative, for any $x \in\left[x_{0}, x_{N}\right]$ and $k \in\{1,2, \ldots, N\}$, $\left(D_{x_{0}}^{v} f\right)\left(L_{k}(x)\right)=\frac{d^{n}}{d\left(L_{k}(x)\right)^{n}}\left(I_{x_{0}}^{n-v} f\right)\left(L_{k}(x)\right)$.Replacing $v$ by $n-v$ in(6) and substituting above, we have

$$
\begin{aligned}
\left(D_{x_{0}}^{v} f\right)\left(L_{k}(x)\right) & =\frac{d^{n}}{d\left(L_{k}(x)\right)^{n}}\left\{a_{k}^{n-v} I_{x_{0}}^{n-v}\left(\gamma_{k} f(x)+q_{k}(x)\right)+\frac{1}{\Gamma(n-v)}\left[\int_{x_{0}}^{x_{k}-1}\left(L_{k}(x)-t\right)^{n-v-1} f(t) d t\right]\right\} \\
& =a_{k}^{-v} D_{x_{0}}^{v}\left(\gamma_{k} f(x)+D_{x_{0}}^{v} q_{k}(x)\right)+\frac{1}{\Gamma(n-v)} \frac{d^{n}}{d\left(L_{k}(x)\right)^{n}}\left[\int_{x_{0}}^{x_{k-1}}\left(L_{k}(x)-t\right)^{n-v-1} f(t) d t\right] \\
& =a_{k}^{-v} D_{x_{0}}^{v}\left(\gamma_{k} f(x)+D_{x_{0}}^{v} q_{k}(x)\right)+\frac{1}{\Gamma(n-v)}\left(\prod_{j=1}^{n} n-j-v\right)\left[\int_{x_{0}}^{x_{k-1}}\left(L_{k}(x)-t\right)^{-v-1} f(t) d t\right]
\end{aligned}
$$


Since $\gamma_{k}<a_{k}^{v}$ it implies that $\left|a_{k}^{-v} \gamma_{k}\right|<1$. Using (7), it is easily seen that $\left(D_{x_{0}}^{v} f\right)\left(L_{k}(x)\right)=a_{k}^{-v} \gamma_{k} D_{x_{0}}^{v} f(x)+$ $q_{k}^{d v}(x)$ and hence the Riemann-Liouville fractional derivative of a FIF of order $v$ is also a FIF. $\square$

\section{Blancmange function}

In this section, we shall first show that the Blancmange function or otherwise called the Takagi fractal curve is a Fractal Interpolation Function for appropriate interpolation points. Then, we shall derive the fractional integral of order $\boldsymbol{v}$ of Blancmange Curve using the results from Section 3. Finally, we show that the fractional derivative of Blancmange Curve is not a FIF for any value of $v$.

The Blancmange Curve (Figure 1) is defined on $[0,1]$ as $\boldsymbol{B}(\boldsymbol{x})=\sum_{n=\mathbf{0}}^{\infty} \frac{s\left(2^{n} x\right)}{2^{n}} \boldsymbol{x} \in[\mathbf{0}, \mathbf{1}]$, where, $\boldsymbol{s}(\boldsymbol{y})=$ $\min _{\boldsymbol{m} \in \mathbb{Z}}|\boldsymbol{y}-\boldsymbol{m}|, \boldsymbol{y} \in \mathbb{R}$. It is observed that the Blancmange function satisfies the recursive equation given by

$$
B\left(\frac{x+k-1}{2}\right)=\frac{1}{2} B(x)+\frac{k-1+(-1)^{k-1} x}{2}, \quad x \in[0,1], \text { for } k=1,2 .
$$

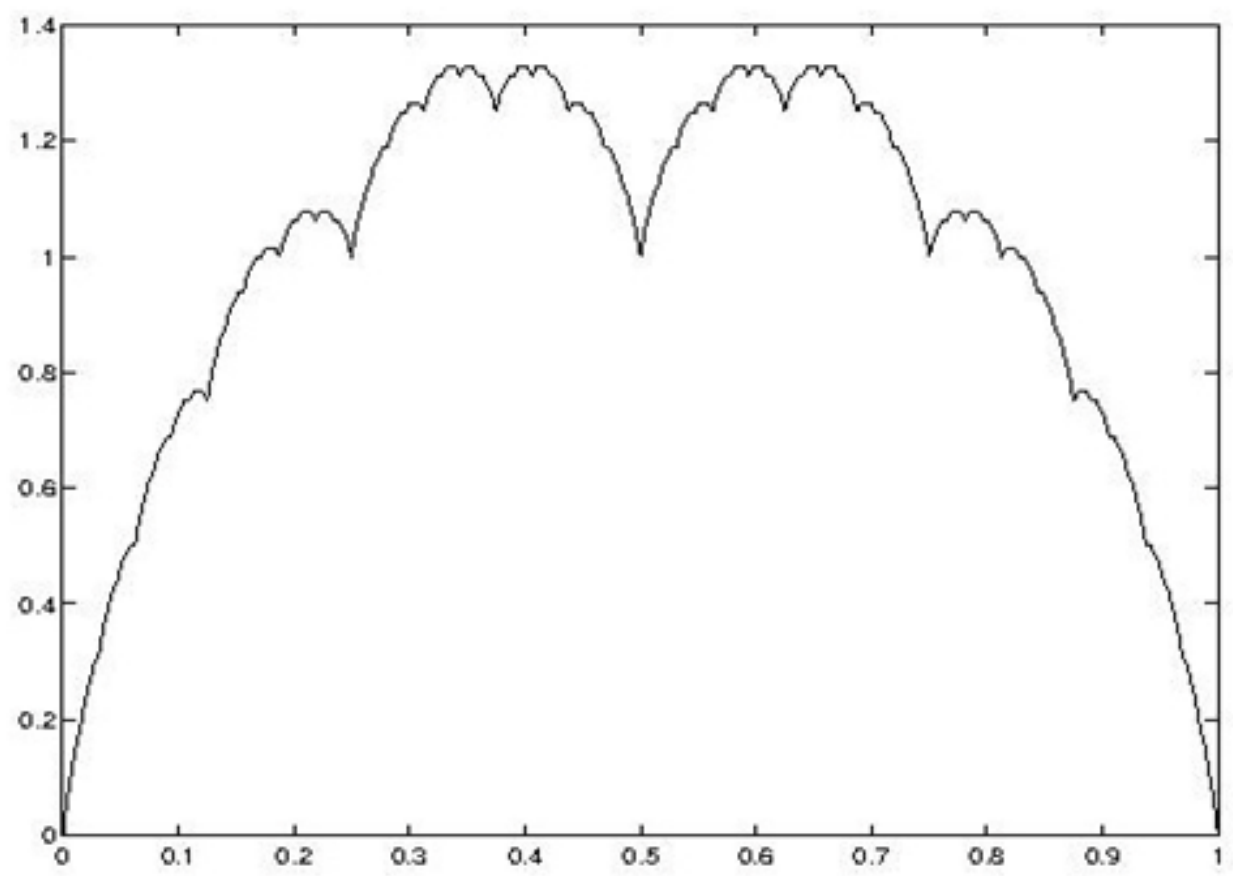

Figure 1. Blancmange Curve or Takagi Curve.

Theorem 1: Blancmange Curve is a Fractal Interpolation Function on [0, 1].

Proof: Consider $\Lambda=\left\{(\mathbf{0}, \mathbf{0}),\left(\frac{1}{2}, \frac{1}{2}\right),(\mathbf{1}, \mathbf{0})\right\}$. Let $\boldsymbol{I}=[\mathbf{0}, \mathbf{1}]$. Then, the IFS required to construct the Blancmange Curve is given by $\left\{I \times \mathbb{R} ; \boldsymbol{\omega}_{k}, \boldsymbol{k}=\mathbf{1}, 2\right\}$ where, $\boldsymbol{\omega}_{k}(x, y)=\left(\frac{x+k-1}{2}, \frac{1}{2} y+\frac{k-1+(-1)^{k-1} x}{2}\right)$. It is easily seen that the above IFS is hyperbolic, and the attractor of the above IFS is a graph of a continuous function that satisfies the recursive equation given by (8). Hence, by the uniqueness of FIF, the Blancmange curve is a Fractal Interpolation Function on $[0,1]$ constructed from the interpolation data given by $\boldsymbol{\Lambda}$ and using the IFS given above.

Now, we shall describe the Riemann-Liouville fractional integral of order $v$ of the Blancmange curve defined on $[0,1]$.

Theorem 2: Riemann-Liouville fractional integral of order $v$ of Blancmange curve defined on $[0,1]$ is a FIF passing through the data $\left\{\left(x_{i}, y_{i}^{v}\right) \in \mathbb{R}^{\wedge} 2: i=0,1,2\right\}$, where $y_{0}^{v}=0, y_{1}^{v}=\frac{v+2}{2^{v+1} \Gamma(v+2)}$ and $y_{2}^{v}=\frac{1}{v+1}$.

Proof: For $k=1,2$, define $F_{k}^{v}(x, y)=\frac{1}{2^{v+1}} y+q_{k}^{v}(x)$ where, $q_{k}^{v}(x)=\frac{x^{v}}{2^{v+1} \Gamma(1+v)}\left[(k-1)+\frac{(-1)^{k-1} x}{1+v}\right]+$ $\frac{1}{\Gamma(v)} \int_{x_{0}}^{x_{k-1}}\left(\frac{x+k-1}{2}-t\right)^{v-1} B(t) d t$. It is observed that $F_{1}^{v}\left(1, \frac{1}{\Gamma(v+1)}\right)=\frac{1}{2^{v+1} \Gamma(1+v)}+\frac{1}{2^{v+1} \Gamma(2+v)}=F_{2}^{v}(0,0)$. Therefore, by Proposition 1, the v-order Riemann-Liouville fractional integral of Blancmange curve is also a FIF, and it satisfies the recursive equation 


$$
\begin{aligned}
I^{v} B\left(\frac{x+k-1}{2}\right) & =\frac{1}{2^{v+1}} I^{v} B(x)+\frac{x^{v}}{2^{v+1} \Gamma(1+v)}\left[(k-1)+\frac{(-1)^{k-1} x}{1+v}\right]+\frac{1}{\Gamma(v)} \int_{x_{0}}^{x_{k-1}}\left(\frac{x+k-1}{2}-t\right)^{v-1} B(t) d t \\
& =\frac{1}{2^{v+1}} I^{v} B(x)+q_{k}^{v}(x)
\end{aligned}
$$

Thus, Riemann-Liouville fractional integral of order $v$ of Blancmange curve defined on $[0,1]$ is a FIF passing through the data $\left\{\left(\boldsymbol{x}_{\boldsymbol{i}}, \boldsymbol{y}_{\boldsymbol{i}}^{\boldsymbol{v}}\right) \in \mathbb{R}^{\wedge} \mathbf{2}: \boldsymbol{i}=\mathbf{0}, \mathbf{1}, \mathbf{2}\right\}$.

Having described that the Riemann-Liouville fractional integral of the Blancmange curve of order $v$ is a FIF, it is natural to seek the Riemann-Liouville fractional derivative of Blancmange curve of order $v$ to be a FIF as earlier. However, we shall show that the Riemann-Liouville fractional derivative of the Blancmange curve defined on [0, 1] is not a FIF for any $v$.

Theorem 3: Riemann-Liouville fractional derivative of the Blancmange curve defined on [0, 1] is not a FIF for any $v$.

Proof: By Proposition 2, the v-order Riemann-Liouville fractional derivative of Blancmange curve is a FIF provided $\gamma_{\boldsymbol{k}}<\boldsymbol{a}_{\boldsymbol{k}}^{\boldsymbol{v}}$ and equation (7) is satisfied. Since $\boldsymbol{a}_{\boldsymbol{k}}=\boldsymbol{\gamma}_{\boldsymbol{k}}=\frac{\mathbf{1}}{\mathbf{2}}$, the value of $v$ can lie only between $\mathbf{0}$ and $\mathbf{1}$. Therefore, for $0<v<1, D^{v} q_{k}(x)=\frac{d}{d x}\left[\frac{1}{\Gamma(1-v)} \int_{0}^{x}(x-t)^{-v} q_{k}(t) d t\right]=\frac{-v}{\Gamma(1-v)} \int_{0}^{x}(x-t)^{-v-1} q_{k}(t) d t$. Now, putting $\boldsymbol{q}_{k}(\boldsymbol{t})=\frac{\boldsymbol{k - 1 + ( - 1 ) ^ { k - 1 } t}}{2}$ for $t \in[0,1]$, we see that

$$
D^{v} q_{k}(x)=\frac{-v}{\Gamma(1-v)} \int_{x_{0}}^{x}(x-t)^{-v-1}\left[\frac{k-1+(-1)^{k-1} t}{2}\right] d t=\frac{1}{2 \Gamma(1-v)}\left\{(k-1) x^{-v}+(-1)^{k-1} \frac{x^{1-v}}{(1-v)}\right\}
$$

As $\boldsymbol{x} \rightarrow \boldsymbol{0}$, the function $\boldsymbol{D}^{\boldsymbol{v}} \boldsymbol{q}_{\mathbf{2}}(\boldsymbol{x})$ goes to $\infty$. Hence, the Riemann-Liouville fractional derivative of the Blancmange curve defined on $[0,1]$ is not a FIF for any value of $v$. $\square$

\section{Cantor function}

In this section, we shall first show that the Cantor function or otherwise called the devil's staircase is a Fractal Interpolation Function for appropriate interpolation points. Then, we shall derive the fractional integral of order $\boldsymbol{v}$ of Cantor's function using the results from Section 3. Finally, we show thatthefractional derivative of Cantor function is not a FIF for any value of $v$.

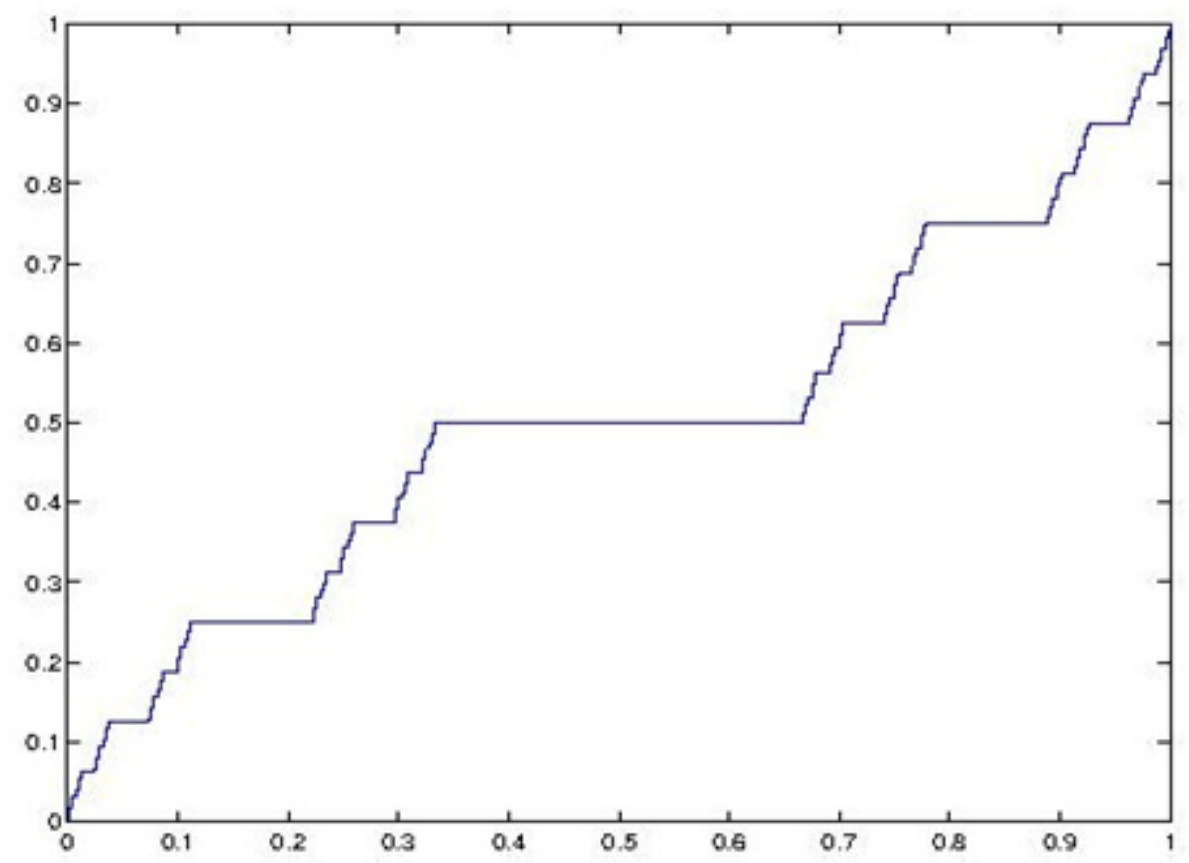

Figure 2. Cantor Function or Devil's Staircase. 
The Cantor function (Figure 2) is defined on $[0,1]$ as $\boldsymbol{C}(\boldsymbol{x})=\lim _{n \rightarrow \infty} \boldsymbol{f}_{\boldsymbol{n}}(\boldsymbol{x}) \boldsymbol{x} \in[\mathbf{0}, \mathbf{1}]$, where,

$$
f_{0}(x)=x, f_{n+1}(x)=\left\{\begin{array}{ll}
0.5 f_{n}(3 x) & \text { if } 0 \leq x \leq \frac{1}{3} \\
0.5 & \text { if } \frac{1}{3}<x<\frac{2}{3} \\
0.5 f_{n}(3 x-2) & \text { if } \frac{2}{3} \leq x \leq 1
\end{array} .\right.
$$

Theorem 4: Cantor function is a Fractal Interpolation Function on $[0,1]$.

Proof: Consider $\Delta=\left\{(\mathbf{0}, \mathbf{0}),\left(\frac{1}{3}, \frac{1}{2}\right),\left(\frac{2}{3}, \frac{1}{2}\right)(\mathbf{1}, \mathbf{0})\right\}$ and $I=[\mathbf{0}, \mathbf{1}]$. Then, the IFS required to construct Cantor function is given by $\left\{I \times I ; \omega_{k}, k=1,2,3\right\}$ where, $\omega_{k}(x, y)=\left(\frac{x+k-1}{3}, \gamma_{k} y+q_{k}(x)\right)$ with $\gamma_{1}=\gamma_{3}=q_{2}(x)=$ $q_{3}(x)=\frac{1}{2}$

and $\gamma_{2}=q_{1}(x)=\mathbf{0}$. It is observed that that the graph of Cantor function is the attractor of the given IFS and satisfies the recursive equation $\boldsymbol{C}\left(\frac{x+\boldsymbol{k}-1}{3}\right)=\gamma_{k} \boldsymbol{C}(x)+\boldsymbol{q}_{k}(x)$. Hence, by the uniqueness of FIF, the Cantor function is a Fractal Interpolation Function on $[0,1]$ constructed from the interpolation data given by $\boldsymbol{\Delta}$ and using the given IFS. $\square$

Now, we shall describe the Riemann-Liouville fractional integral of order $v$ of Cantor function defined on $[0,1]$.

Theorem 5: Riemann-Liouville fractional integral of order $v$ of Cantor function defined on $[0,1]$ is a FIF passing through the data $\left\{\left(x_{i}, y_{i}^{v}\right) \in \mathbb{R}^{2}: i=\mathbf{0}, \mathbf{1}, 2,3\right\}$, where $y_{0}^{v}=\mathbf{0}, y_{1}^{v}=\frac{1}{2 \cdot\left(2.3^{v}-1\right) \Gamma(v+1)}, y_{2}^{v}=\frac{2^{v-1}}{3^{v} \Gamma(v+1)}$ and $y_{3}^{v}=$ $\frac{3^{v}}{\left(2.3^{v}-1\right) \Gamma(v+1)}$.

Proof: For $k=1,2,3$, define $F_{k}^{v}(x, y)=\gamma_{k}^{v} y+q_{k}^{v}(x)$ where, $\gamma_{1}^{v}=\gamma_{3}^{v}=\frac{1}{2.3^{v}}, \gamma_{2}^{v}=q_{1}^{v}=0$, and

$$
q_{k}^{v}(x)=\frac{(x+(k-2))^{v}}{2 \times 3^{v} \Gamma(1+v)}+\frac{1}{\Gamma(v)} \int_{0}^{\frac{1}{3}}\left(\frac{x+k-1}{2}-t\right)^{v-1} C(t) d t, k=2,3 .
$$

It is observed that $\boldsymbol{F}_{1}^{v}\left(\mathbf{1}, \frac{3^{v}}{\left(2.3^{v}-1\right) \Gamma(v+1)}\right)=\frac{\mathbf{1}}{2 .\left(2.3^{v}-1\right) \Gamma(v+1)}=\boldsymbol{q}_{k}^{v}(\mathbf{0})=\boldsymbol{F}_{2}^{v}(\mathbf{0}, \mathbf{0})$ and $\boldsymbol{F}_{2}^{v}\left(\mathbf{1}, \frac{3^{v}}{\left(2.3^{v}-1\right) \Gamma(v+1)}\right)=$ $\frac{1}{2 .\left(2.3^{v}-1\right) \Gamma(v+1)}=q_{3}^{v}(0)=F_{3}^{v}(\mathbf{0}, 0)$. Therefore, by Proposition 1, the $v$-order Riemann-Liouville fractional integral of Cantor function is also a FIF, and it satisfies the recursive equation $I^{v} \boldsymbol{C}\left(\frac{x+\boldsymbol{k}-\mathbf{1}}{3}\right)=\gamma_{\boldsymbol{k}} \boldsymbol{I}^{v} \boldsymbol{C}(\boldsymbol{x})+\boldsymbol{q}_{\boldsymbol{k}}^{v}(\boldsymbol{x})$. Thus, Riemann-Liouville fractional integral of order $v$ of Cantor function defined on $[0,1]$ is a FIF passing through the data $\left\{\left(x_{i}, y_{i}^{v}\right) \in \mathbb{R}^{3}: i=0,1,2,3\right\}$.

Having described that the Riemann-Liouville fractional integral of Cantor function of order $v$ is a FIF, it is natural to seek Riemann-Liouville fractional derivative of Cantor function of order $v$ to be a FIF as earlier. However, we shall show that the Riemann-Liouville fractional derivative of Cantor function defined on $[0,1]$ is also not a FIF for any $v$. $\square$

Theorem 6: Riemann-Liouville fractional derivative of Cantor function defined on $[0,1]$ is not a FIF for any $v$.

Proof: By Proposition 2, the v-order Riemann-Liouville fractional derivative of Cantor function is a FIF if $\boldsymbol{\gamma}_{\boldsymbol{k}}<\boldsymbol{a}_{\boldsymbol{k}}^{\boldsymbol{v}}$ and equation (7) is satisfied. Since $a_{k}=\frac{1}{3}$, and $\gamma_{1}=\gamma_{3}=\frac{1}{2}, \gamma_{2}=\mathbf{0}$. the value of $v$ can lie only between 0 and $\frac{\log 2}{\log 3}$. Therefore, for $0<v<\frac{\log 2}{\log 3}<1, D^{v} q_{k}(x)=\frac{d}{d x}\left[\frac{1}{\Gamma(1-v)} \int_{0}^{x}(x-t)^{-v} q_{k}(t) d t\right]=\frac{-v}{\Gamma(1-v)} \int_{0}^{x}(x-t)^{-v-1} q_{k}(t) d t$.

Now, putting $q_{2}(t)=q_{3}(t)=\frac{1}{2}$ for $t \in[0,1]$, we see that $D^{v} q_{k}(x)=\frac{-v}{\Gamma(1-v)} \int_{0}^{x}(x-t)^{-v-1}\left[\frac{1}{2}\right] d t=\frac{x^{-v}}{2 \Gamma(1-v)}$.

As $\boldsymbol{x} \rightarrow \mathbf{0}$, the function $\boldsymbol{D}^{v} \boldsymbol{q}_{2}(\boldsymbol{x})$ and $\boldsymbol{D}^{v} \boldsymbol{q}_{3}(\boldsymbol{x})$ goes to $\infty$. Hence, the Riemann-Liouville fractional derivative of the Cantor function defined on $[0,1]$ is not a FIF for any value of $v$. $\square$

\section{Conclusion}

In this paper, Riemann-Liouville fractional calculus of Blancmange Curve and Cantor Functions are studied. It is first shown that Blancmange Curve and Cantor Function defined on the interval $[0,1]$ is a Fractal Interpolation Function for appropriate interpolation points. Then, using the properties of FIF, it is proved that Riemann-Liouville fractional integral of order $v$ of Blancmange Curve and Cantor Function is a FIF. However, the Riemann-Liouville fractional derivative of Blancmange Curve and Cantor Function is shown to be not a FIF for any value of $v$. 


\section{References}

[1] Barnsley, M. F. (1986). Fractal functions and interpolation. Constructive Approximation, 2, 303-329.

[2] Barnsley, M. F., Elton, J., Hardin, D., and Massopust, P. (1989). Hidden variable fractal interpolation functions. SIAM Journal of Mathematical Analysis, 20(5), 1218-1242.

[3] Chand, A. K. B. and Kapoor, G. P. (2007). Smoothness analysis of coalescence hidden variable fractal interpolation functions. International Journal of Non-Linear Science, 3, 15-26.

[4] Kapoor, G. P. and Prasad, S. A. (2014). Multiresolution analysis based on coalescence hidden-variable fractal interpolation functions. International Journal of Computational Mathematics, Article ID 531562.

[5] Navascués, M. A. and Sebastián, M. V. (2004). Generalization of Hermite functions by fractal interpolation. Journal of Approximation Theory, 131, 19-29.

[6] Kapoor, G. P. and Prasad, S. A. (2014). Convergence of cubic spline super fractal interpolation functions. Fractals: Complex Geometry, Patterns, and Scaling in Nature and Society, 22(1), 1-7.

[7] Kapoor, G. P. and Prasad, S. A. (2015). Super fractal interpolation functions. International Journal of Non-Linear Science, 19(1), 20-29.

[8] Kempfle, S. and Schaefer, I. (1999). Functional Calculus Method Versus Riemann-Liouville Approach. Fractional Calculus and Applied Analysis, 2(4), 415-428.

[9] Changpin Li, Deliang Qian, and Yang Quan Chen. (2011). On Riemann-Liouville and Caputo Derivatives. Discrete Dynamics in Nature and Society, Article ID 562494.

[10] Edmundo Capelas de Oliveira and José António Tenreiro Machado. (2014). A Review of Definitions for Fractional Derivatives and Integral. Mathematical Problems in Engineering, Article ID: 238459.

[11] Hilfer, R. (2008). Threefold Introduction to Fractional Derivatives, In. Anomalous Transport: Foundations and Applications, Wiley-VCH, Weinheim.

[12] Huo-Jun Ruan, Wei-Yi Su, and Kui Yao. (2009). Box dimension and fractional integral of linear fractal interpolation functions. Journal of Approximation Theory, 161, 187-197.

[13] XueZai Pan. (2014). Fractional Calculus of Fractal Interpolation Function on [0; b]. Abstract and Applied Analysis, Article ID: 640628.

[14] Prasad, S. A. (2017). Fractional calculus of coalescence hidden-variable fractal interpolation functions. Fractals: Complex Geometry, Patterns, and Scaling in Nature and Society, 25(2), Article ID 1750019. 